\title{
GSK-3 $\alpha$ directly regulates $\beta$-adrenergic signaling and the response of the heart to hemodynamic stress in mice
}

\author{
Jibin Zhou, ${ }^{1}$ Hind Lal, ${ }^{1}$ Xiongwen Chen, ${ }^{2}$ Xiying Shang, ${ }^{1}$ Jianliang Song, ${ }^{1}$ Yingxin Li, ${ }^{2}$ \\ Risto Kerkela, ${ }^{1}$ Bradley W. Doble, ${ }^{3}$ Katrina MacAulay, ${ }^{4}$ Morgan DeCaul, ${ }^{1}$ \\ Walter J. Koch,, ${ }^{1}$ John Farber, ${ }^{1}$ James Woodgett, ${ }^{4}$ Erhe Gao, ${ }^{1}$ and Thomas Force ${ }^{1}$ \\ ${ }^{1}$ Thomas Jefferson University, Philadelphia, Pennsylvania, USA. ${ }^{2}$ Temple University School of Medicine, Philadelphia, Pennsylvania, USA. \\ ${ }_{3}^{3}$ McMaster Stem Cell and Cancer Research Institute, McMaster University, Hamilton, Ontario, Canada. \\ ${ }^{4}$ Samuel Lunenfeld Research Institute, Mount Sinai Hospital, Toronto, Ontario, Canada.
}

\begin{abstract}
The glycogen synthase kinase-3 (GSK-3) family of serine/threonine kinases consists of 2 highly related isoforms, $\alpha$ and $\beta$. Although GSK-3 $\beta$ has an important role in cardiac development, much remains unknown about the function of either GSK-3 isoform in the postnatal heart. Herein, we present what we believe to be the first studies defining the role of GSK-3 $\alpha$ in the mouse heart using gene targeting. Gsk $3 a^{-/-}$mice over 2 months of age developed progressive cardiomyocyte and cardiac hypertrophy and contractile dysfunction. Following thoracic aortic constriction in young mice, we observed enhanced hypertrophy that rapidly transitioned to ventricular dilatation and contractile dysfunction. Surprisingly, markedly impaired $\beta$-adrenergic responsiveness was found at both the organ and cellular level. This phenotype was reproduced by acute treatment of WT cardiomyocytes with a small molecule GSK-3 inhibitor, confirming that the response was not due to a chronic adaptation to LV dysfunction. Thus, GSK-3 $\alpha$ appears to be the central regulator of a striking range of essential processes, including acute and direct positive regulation of $\beta$-adrenergic responsiveness. In the absence of GSK-3 $\alpha$, the heart cannot respond effectively to hemodynamic stress and rapidly fails. Our findings identify what we believe to be a new paradigm of regulation of $\beta$-adrenergic signaling and raise concerns given the rapid expansion of drug development targeting GSK-3.
\end{abstract}

\section{Introduction}

The glycogen synthase kinase-3 (GSK-3) family consists of 2 isoforms, $\alpha$ and $\beta$, which are $98 \%$ identical within their kinase domains but differ substantially in their $\mathrm{N}$ - and C-terminal sequences (1). Unlike most protein kinases, GSK-3 is typically active in unstimulated cells and is inhibited in response to a variety of inputs (1). Since GSK-3-mediated phosphorylation of substrates usually leads to inhibition of those substrates, the end result of stimulus-induced inhibition of GSK-3 is typically activation of its downstream targets. Innumerable factors have been reported to be bona fide substrates of GSK-3 $(2,3)$.

Most of the attention on this family has centered on GSK-3 $\beta$, which, since its discovery, has been reported to regulate an astonishing variety of cellular processes. However, this bias toward GSK-3 $\beta$ as the dominant isoform may go back many years to studies in which mammalian GSK-3 $\beta$ was better able than GSK-3 $\alpha$ to rescue the frizzled phenotype in Drosophila, which resulted from a mutation in fly GSK-3 (Zw3/shaggy) (4, 5). In fact, recent studies done in ES cells in which Gsk3a, Gsk3b, or both have been deleted demonstrate that the 2 isoforms are entirely redundant in terms of regulating the canonical Wnt pathway (6). Thus, not until 3 out of 4 alleles of Gsk3a and Gsk3b are deleted is there any spontaneous activation of Wnt $/ \beta$-catenin signaling, and not until all 4 are deleted is the activation marked.

However, GSK-3s are clearly not redundant in all functions. For example, GSK- $3 \alpha$ is not able to compensate for loss of GSK-3 $\beta$ during cardiac development (7). Indeed, the hearts of embryos in

Conflict of interest: The authors have declared that no conflict of interest exists. Citation for this article: J Clin Invest. 2010;120(7):2280-2291. doi:10.1172/JCI41407. which Gsk3b has been deleted were characterized by a hypertrophic myopathy that was due primarily to hyperproliferation of cardiomyocytes (7). This led to near obliteration of the RV and LV cavities and, together with structural defects, including double outlet RV and ventricular septal defect, common congenital abnormalities in humans, uniformly led to late embryonic or immediate postnatal death. Furthermore, based on studies in ES cells in which one or the other isoform has been deleted, GSK-3 $\beta$ appears to promote cardiomyocyte differentiation, whereas GSK- $3 \alpha$ plays a much more minor role $(3,7)$.

Although the above findings clearly demonstrate that GSK- $3 \beta$ plays a dominant role in cardiac development, the role of the isoforms in the postnatal heart is not known. This is due to the fact that all studies to date have used transgenesis, knockin of activated mutants, or nonselective small molecule inhibitors (e.g., BIO) $(8-14)$. None of these strategies allow one to define the true biology of GSK-3s or an understanding of isoform-specific effects.

Not surprisingly, some of these studies have reached seemingly contradictory conclusions, particularly concerning the role of GSK- $3 \alpha$. For example, work from the same laboratory alternately reported that constitutively active GSK-3 $\alpha$ suppressed pressure overload-induced hypertrophy in a transgenic model but enhanced hypertrophy in a knockin model $(13,14)$. Further complicating understanding, the strategy used in these models (mutation of the inhibitory serine 21 phosphorylation site of GSK-3 $\alpha$ and serine 9 phosphorylation site of GSK-3 $\beta$ to alanine residues) blocks only 1 mechanism by which GSK- $3 \alpha$ can be inhibited (i.e., PI3-kinase-mediated activation of Akt) and does not affect Wntmediated or p38-MAPK-mediated inhibition (15-17). Finally, to 
A

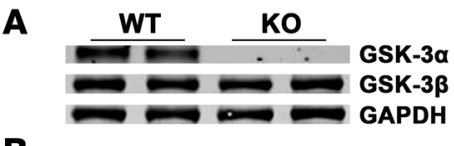

B

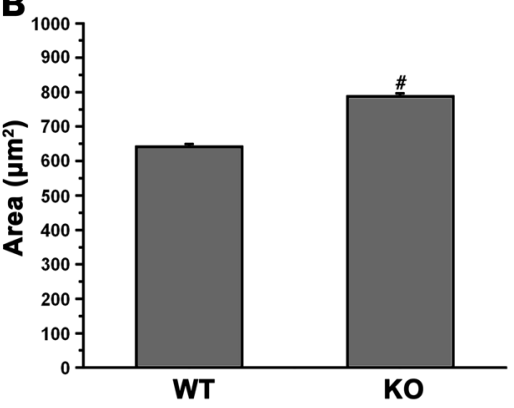

C

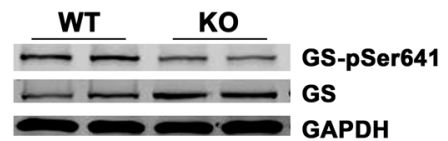

D

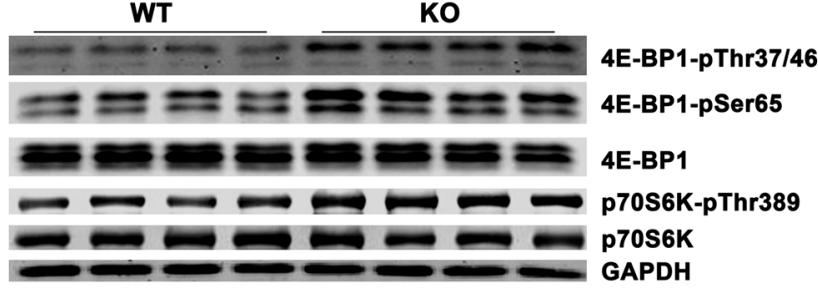

Figure 1

Deletion of Gsk3a leads to cardiac hypertrophy. (A) Deletion of Gsk3a does not lead to compensatory upregulation of GSK-3 $\beta$. Immunoblot of

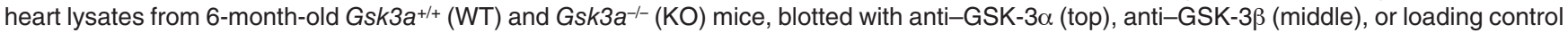
(anti-GAPDH, bottom). (B) Cardiomyocyte size determination. Cardiomyocytes were isolated from 6-month-old KO and WT mice, as described in Methods. Cell area was then determined in $551 \mathrm{KO}$ myocytes from mice $(n=6)$ and 576 WT myocytes from mice $(n=6)$, as described in Methods. ${ }^{\#} P<0.01$ versus WT. (C) GSK-3 $\alpha$ regulates phosphorylation of glycogen synthase, Ser641. Mice were fasted overnight, and then heart lysates were immunoblotted with anti-phospho-glycogen synthase (GS-pSer641), anti-glycogen synthase (GS), or GAPDH. The image is representative of findings from WT $(n=6)$ and KO $(n=6)$ mice. (D) Dysregulation of mTORC1 in the KO mice. Lysates of hearts from 4-month-old WT and KO mice were immunoblotted for (top to bottom) phospho-4E-BP1 Thr37/46, phospho-4E-BP1 Ser65, total 4E-BP1, phospho-p70S6K Thr389, total p70S6K, or GAPDH. Note the increase in phosphorylation of 4E-BP1 at both sites and of p70S6K in the KO.

date, no studies have examined possible metabolic effects of GSK3 s and how they might affect cardiac function, even though this is a critical factor in GSK-3-regulated disease processes, including glucose intolerance and diabetes $(18,19)$.

Herein, we used a mouse in which Gsk3a has been deleted (KO) to define its roles in the heart. We found critical roles for this kinase in cardiac growth, pathologic hypertrophy, maintenance of mitochondrial integrity, and regulation of contractile function. Most importantly, we found that the response to $\beta$-adrenergic stimulation, which is one of the most critical responses in the heart to stress, if not the most important one, depends on GSK-3 $\alpha$. In the absence of GSK-3 $\alpha$, there is a gradual deterioration in LV function that is dramatically accelerated when the heart is exposed to hemodynamic stress.

\section{Results}

Hypertrophy in the GSK-3 $\alpha$ KO. The creation of the GSK-3 $\alpha \mathrm{KO}$ mouse was previously described $(6,20)$. Briefly, a pCAGGS (chicken $\beta$-actin) Cre deleter mouse strain generated by Nagy (21), which expresses Cre in all tissues, was crossed with the Gsk3a floxed mouse (exon 2) to generate the KO mice. Subsequently, the Cre transgene was bred out, and Cre was no longer detectable by RT-PCR in the mice used herein. Importantly, there was no compensatory upregulation of GSK-3 $\beta$ in the heart following deletion of Gsk3a (Figure 1A). As previously reported, Gsk3a KO mice appeared normal at birth (7). However, beginning after 3 months of age, cardiac hypertrophy developed (Tables 1 and 2). Cardiac hypertrophy was due, at least in part, to cardiomyocyte hypertrophy, since cardiomyocyte size was significantly increased in the $\mathrm{KO}$ mice (Figure 1B).

The hypertrophy was not due to hypertension, since LV systolic pressures (LVSPs) were not increased in the KO mice at up to 6 months of age (see below). The hypertrophy was also not likely due to volume overload, since LV end-diastolic dimension (EDD), as assessed by echocardiography, was not increased in the $\mathrm{KO}$ mice at up to 6 months of age (Table 2) and LV end-diastolic pressure was not increased in the $\mathrm{KO}$ mice until 6 months of age (data not shown).

Given the key role of GSK-3 in regulating glycogen storage, we asked whether enhanced glycogen storage, which leads to cardiac hypertrophy in patients with mutations in the $\gamma_{2}$ subunit of AMPK (22), could account for the hypertrophy in the Gsk3a-/- mice. Although we did find that GSK-3 $\alpha$ regulated phosphorylation status of the critical Ser641 residue of glycogen synthase (Figure 1C) and, consequently, glycogen storage in the heart, the absolute amount of glycogen in the heart was small $(0.05 \%$ of that in the liver), and the increase compared with WT littermates was minimal ( $12.5 \pm 0.36$ vs. $15.7 \pm 0.95 \mathrm{mg} / \mathrm{g}$ of tissue; $P=0.06)$. Thus, increased glycogen storage did not account for the hypertrophy.

We then turned to signaling pathways downstream of GSK-3 that regulate hypertrophic growth. One of these is mTOR complex 1 (mTORC1), the master regulator of protein translation

Table 1

Gravimetric data for the Gsk3a knockout mouse heart

\begin{tabular}{|c|c|c|c|c|}
\hline & $n$ & BW $(g)$ & HW (mg) & HW/BW ratio \\
\hline \multicolumn{5}{|c|}{2 months old } \\
\hline WT & 23 & $26.8 \pm 3.36$ & $102.4 \pm 10.96$ & $3.88 \pm 0.128$ \\
\hline KO & 25 & $27.5 \pm 3.13$ & $107.9 \pm 13.33$ & $3.97 \pm 0.144$ \\
\hline \multicolumn{5}{|c|}{4 months old } \\
\hline WT & 11 & $32.7 \pm 1.96$ & $137.9 \pm 11.94$ & $3.96 \pm 0.290$ \\
\hline KO & 14 & $35.8 \pm 2.56$ & $169.9 \pm 8.25^{A}$ & $4.84 \pm 0.249^{A}$ \\
\hline \multicolumn{5}{|c|}{6 months old } \\
\hline WT & 10 & $35.6 \pm 2.04$ & $146.3 \pm 14.92$ & $4.10 \pm 0.273$ \\
\hline KO & 21 & $37.8 \pm 2.22$ & $184.7 \pm 16.19 \mathrm{~A}$ & $4.89 \pm 0.129 \mathrm{~A}$ \\
\hline
\end{tabular}

Values are mean $\pm \mathrm{SD}$. ${ }^{\mathrm{A} P}<0.05, \mathrm{KO}$ versus $\mathrm{WT}$ mice. 
Table 2

Echocardiographic data for the Gsk3a knockout mouse heart

\begin{tabular}{|c|c|c|c|c|c|c|}
\hline & $n$ & LV mass (mg) & LVPW;d (mm) & EDD (mm) & ESD (mm) & FS (\%) \\
\hline \multicolumn{7}{|c|}{2 months old } \\
\hline WT & 18 & $109.9 \pm 11.15$ & $0.80 \pm 0.065$ & $4.00 \pm 0.245$ & $2.71 \pm 0.210$ & $30.12 \pm 3.066$ \\
\hline KO & 17 & $111.7 \pm 17.05$ & $0.82 \pm 0.075$ & $4.04 \pm 0.341$ & $2.71 \pm 0.395$ & $29.97 \pm 4.640$ \\
\hline \multicolumn{7}{|c|}{4 months old } \\
\hline WT & 18 & $130.2 \pm 15.74$ & $0.83 \pm 0.092$ & $4.18 \pm 0.230$ & $2.66 \pm 0.286$ & $30.89 \pm 3.399$ \\
\hline KO & 17 & $150.7 \pm 26.18^{B}$ & $0.94 \pm 0.062^{\mathrm{B}}$ & $4.20 \pm 0.294$ & $2.67 \pm 0.398$ & $28.47 \pm 3.291^{A}$ \\
\hline \multicolumn{7}{|c|}{6 months old } \\
\hline WT & 23 & $139.0 \pm 21.00$ & $0.88 \pm 0.087$ & $4.23 \pm 0.317$ & $2.72 \pm 0.352$ & $35.93 \pm 5.011$ \\
\hline KO & 23 & $172.1 \pm 29.11^{B}$ & $1.00 \pm 0.096^{A}$ & $4.30 \pm 0.315$ & $2.98 \pm 0.322^{A}$ & $30.22 \pm 3.534$ \\
\hline
\end{tabular}

LV mass (in $\mathrm{mg}$ ) was calculated on M-mode and 2-dimensional echocardiography. Values are mean $\pm \mathrm{SD}$. LVPW;d, diastolic LV posterior wall; ESD, end-systolic dimension; FS, fractional shortening. ${ }^{A} P<0.05$, ${ }^{B} P<0.01 \mathrm{KO}$ versus WT mice.

that is inhibited following GSK-3-mediated phosphorylation of Tsc2 (23). We found significant activation of mTORC1 based on enhanced phosphorylation of 2 of its substrates, 4E-BP1 and p70S6 kinase (Figure 1D). Thus GSK-3 $\alpha$ is a key negative input into mTORC1 signaling, and loss of GSK-3 $\alpha$ leads to inappropriate activation of mTOR in the heart. In contrast, we saw no activation of other hypertrophic signaling pathways including Akt (data not shown) and p38 MAPK, but did find modest activation of ERK1/2 in the basal state (see below).

Pressure overload bypertrophy in the GSK-3 $\alpha \mathrm{KO}$ mice. We then examined the effect of deletion of Gsk $3 a$ on the response to pressure overload induced by transverse aortic constriction (TAC) at 4 months of age. Mice that underwent TAC had an increase in hypertrophy that was more pronounced in $\mathrm{KO}$ compared with WT mice (Figure 2A). This was associated with increased expression of atrial natriuretic peptide (3.7-fold in $\mathrm{KO}$ mice undergoing TAC $[\mathrm{KO} / \mathrm{TAC}]$ vs. WT mice undergoing TAC $[\mathrm{WT} / \mathrm{TAC}] ; P=0.01)$ and a trend toward increased brain natriuretic peptide (1.5-fold in KO/TAC vs. WT/TAC; $P=0.06$ ).

When we plotted heart weight/body weight (HW/BW) versus LVSP, the KO mice had much higher HW/BW at significantly lower systolic pressures (Figure 2, B and C). This was associated with reduced fractional shortening (Figure 2D) and more markedly reduced maximal first derivative of $L V$ pressure rise $(+d P / d t)$ and fall $(-d P / d t)$ (Figure 2, E and F). Since the degree of TAC was the same in WT and KO mice, the data, taken together, are consistent with an enhanced hypertrophic response and an inability of the heart of the GSK-3 $\alpha$ KO mouse to generate a pressor response to TAC.

Although substantially greater in the $\mathrm{KO}$ mice, the full magnitude of the difference in the hypertrophic response between WT and $\mathrm{KO}$ mice was somewhat difficult to compare in the 4-monthold mice, given the baseline differences in heart weight (Tables 1 and 2 and Figure 2A). Therefore, we used TAC in 2-month-old mice, a time at which there are no differences between WT and KO mice in baseline LV mass (Table 1 and Figure 3A). We then followed the mice with weekly echocardiography for 3 weeks. Within 1 week, calculated LV mass in KO and WT mice started to diverge, and at 2 and 3 weeks after TAC, the differences were statistically significant (Figure 3, A and E). In contrast to the WT mice, fractional shortening progressively fell over the 3 -week period in the $\mathrm{KO}$ mice
(Figure 3F). Furthermore, in contrast to WT mice, EDD increased significantly in the $\mathrm{KO}$ mice at the 3-week time point, consistent with adverse remodeling occurring early after TAC (Figure 3C). This adverse remodeling was again associated with an inability of the $\mathrm{KO}$ mice to generate a pressor response to TAC (Figure 3D). The KO hearts were also notable for significant replacement fibrosis, consistent with cell loss. This was widespread and involved approximately 14\% of the surface area of the LV of the KO hearts (Figure 3F).

Contractile dysfunction in the $\mathrm{KO}$ beart. Not only was there marked LV dysfunction after TAC in the KO heart, modest LV dysfunction was also apparent in the nonstressed $\mathrm{KO}$ heart, as assessed by echocardiography at both 4 and 6 months of age (Table 2). To get a sense of the factors that might contribute to the LV dysfunction, we examined transmission EMs from the hearts of 6-month-old KO mice. We found striking abnormalities of mitochondrial structure, characterized by markedly swollen mitochondria, containing only remnants of cristae (Figure 4, A and B). These findings were relatively widespread and strongly suggested opening of the mitochondrial permeability transition pore. Of note, these findings were not present in the 2-month-old or 4-month-old hearts (data not shown). Thus, the mitochondrial abnormalities likely exacerbated the LV dysfunction seen in the 6-month-old mice, but other processes were also involved, since the mitochondrial abnormalities were not present at the earlier time points.

We were most struck by the inability of the $\mathrm{KO}$ heart to mount a contractile response in the setting of pressure stress, and we explored potential mechanisms. Since the $\beta$-adrenergic system is critical for the heart's response to stress, we examined adrenergic responsiveness in the hearts of the 6 -month-old $\mathrm{KO}$ mice exposed to graded isoproterenol infusion. These studies were done in mice in the basal state (i.e., in the absence of prior TAC). We found a significant reduction in the response of the KO heart to isoproterenol compared with that of the WT heart (Figure 4, $\mathrm{C}-\mathrm{F})$. The LVSP response was reduced, albeit not significantly (Figure 4C), but there was a markedly blunted $+\mathrm{dP} / \mathrm{dt}$ (Figure $4 \mathrm{D})$ and $-\mathrm{dP} / \mathrm{dt}$ (Figure $4 \mathrm{E}$ ) response in the $\mathrm{KO}$ compared with the WT heart. The heart rate response to isoproterenol was also blunted (Figure 4F).

At 2 months of age, despite echocardiographic fractional shortening being normal (Figure 3B and Tables 1 and 2), the contractile responses to $\beta$-adrenergic stimulation (LVSP and $+\mathrm{dP} / \mathrm{dt}$ ) were significantly depressed (Figure 5, A and B). - dP/dt was also reduced (Figure $5 \mathrm{C}$ ). The depression of $\beta$-adrenergic responsiveness was also evident at the single cell level, though it did not reach statistical significance in the myocytes isolated from 2-month-old $\mathrm{KO}$ mice (Figure 6, A and B).

We then explored mechanisms of the impaired response to $\beta$-adrenergic stimulation. We first wanted to rule out the possibility that the alterations in $\beta$-adrenergic responsiveness were a consequence, as opposed to the cause, of the LV dysfunction. That 
A

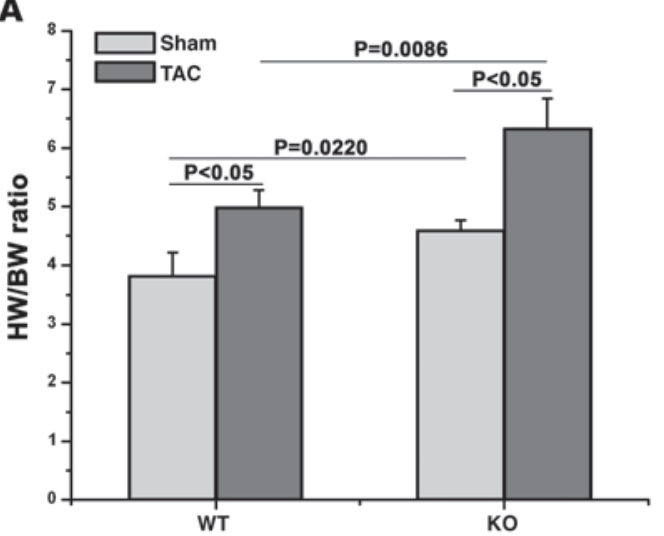

C

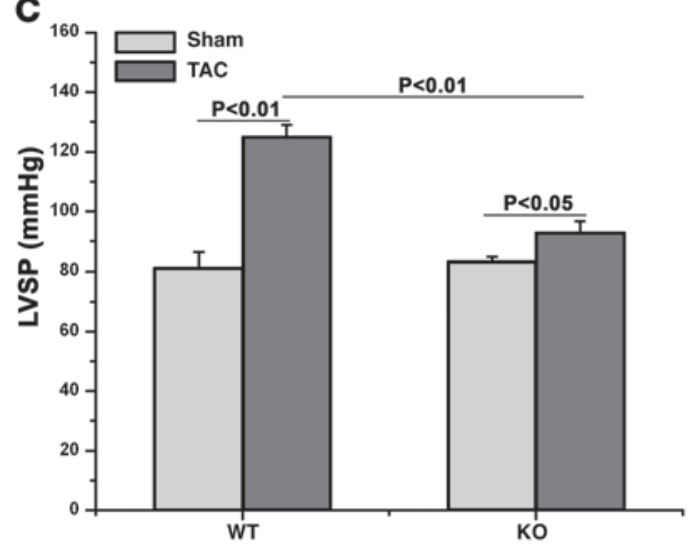

$\mathbf{E}_{1200}$

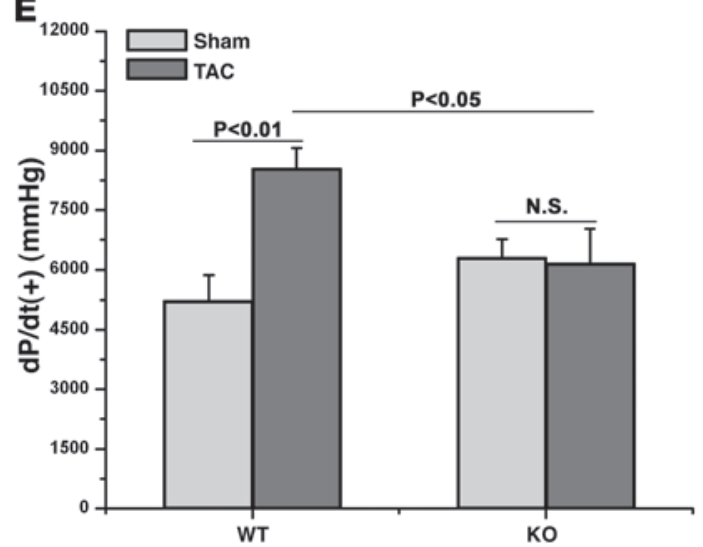

B

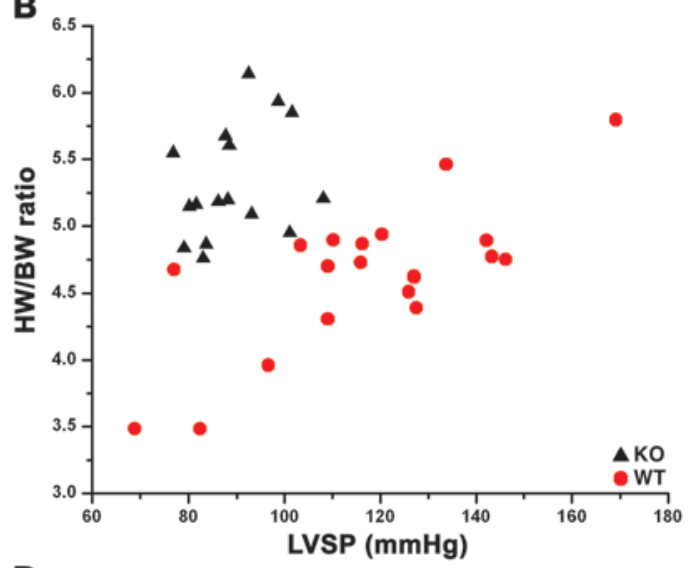

D
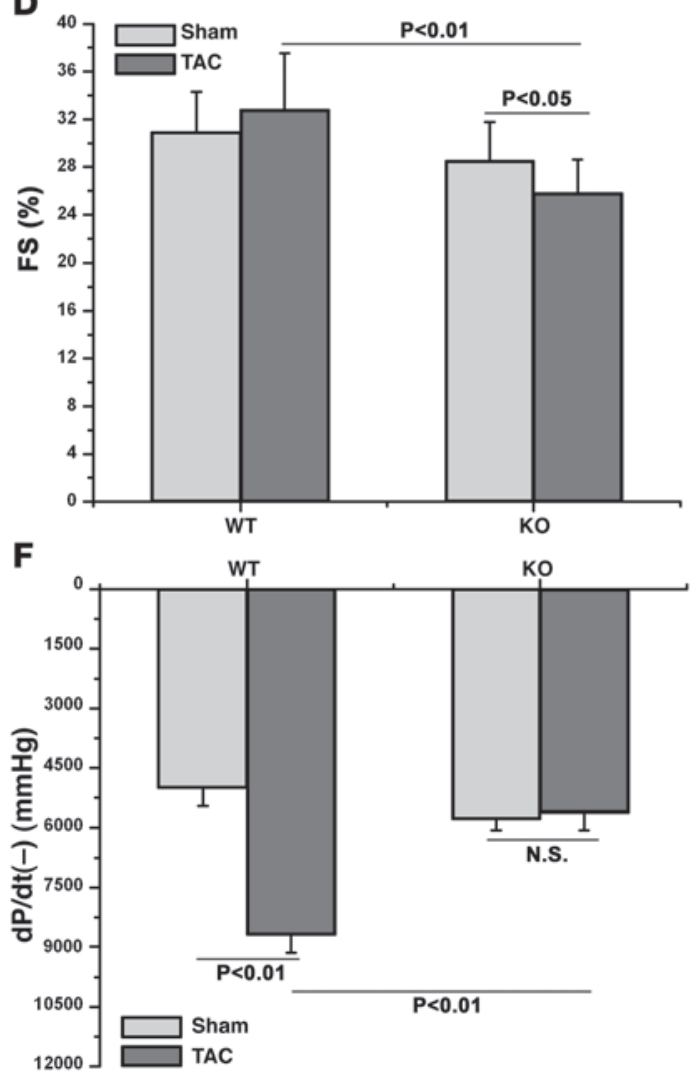

Figure 2

Exaggerated hypertrophy and impaired cardiac function in the 4-month-old KO mouse subjected to pressure stress. Four-month-old WT and KO mice were subjected to TAC or sham TAC for 3 weeks, as described in Methods. (A) At sacrifice, HW/BW ratios (a measure of cardiac hypertrophy) were determined. (B) HW/BW is plotted as the dependent variable, with LVSP as the independent variable. Note the marked increase in HW/BW at all LVSPs for the KO mice (black triangles) when compared with WT mice (red circles). Also note that the KO mice were unable to generate LVSPs of more than $110 \mathrm{mmHg}$. LVSP (C), fractional shortening (FS) (D), $+\mathrm{dP} / \mathrm{dt}(\mathrm{E})$, and $-\mathrm{dP} / \mathrm{dt}(\mathrm{F})$ are shown graphically. Note that all functional parameters are depressed in the KO mice. $n=7 \mathrm{WT}$ and $9 \mathrm{KO}$ sham mice; $n=10 \mathrm{WT} / \mathrm{TAC}$ and $13 \mathrm{KO} / \mathrm{TAC}(\mathbf{A}) ; n=19 \mathrm{WT} / \mathrm{TAC}$ and $16 \mathrm{KO} / \mathrm{TAC}(\mathrm{B}) ; n=6 \mathrm{WT}$ and $7 \mathrm{KO}$ sham mice; $n=10 \mathrm{WT} / \mathrm{TAC}$ and $5 \mathrm{KO} / \mathrm{TAC}(\mathbf{C}-\mathbf{F})$.

is, we wanted to exclude an intrinsic abnormality of the myocyte leading to the decreased responsiveness. Therefore, we treated 4-month-old WT and KO mice with NKH477, a water soluble forskolin derivative that increases cAMP levels in the heart by directly activating adenylyl cyclase (24). Graded infusion of NKH477 markedly increased cAMP levels in the heart, and the increase was com- parable in WT versus KO mice (Figure 7A). Furthermore, cardiac responsiveness to increases in cAMP was equivalent in WT versus $\mathrm{KO}$ mice (Figure 7, B-E), ruling out an intrinsic contractile defect as the cause of the reduced responsiveness in the KO.

We then asked whether the defect in $\beta$-adrenergic responsiveness was due to an impairment in cAMP generation. We 

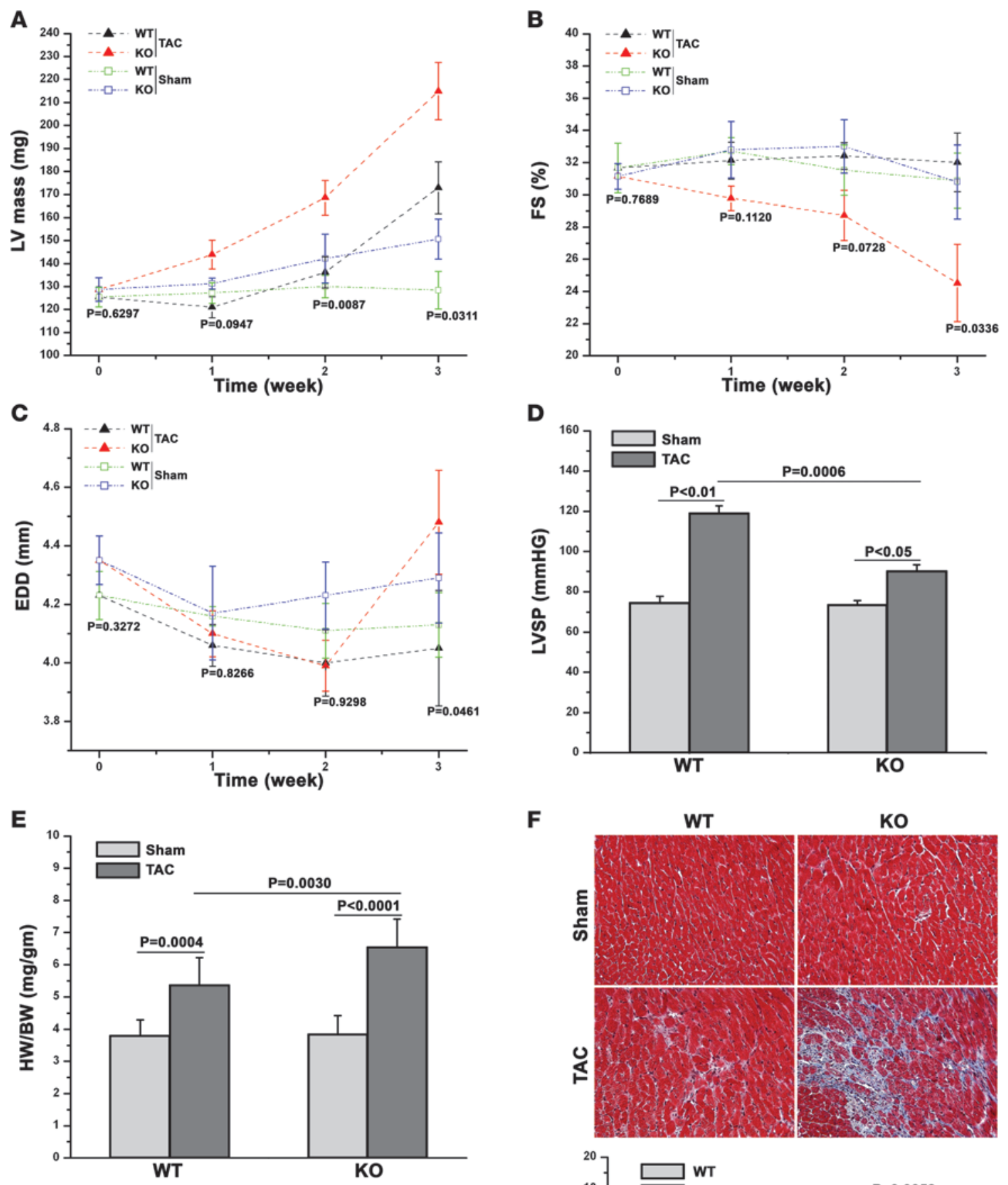

$\mathbf{F}$
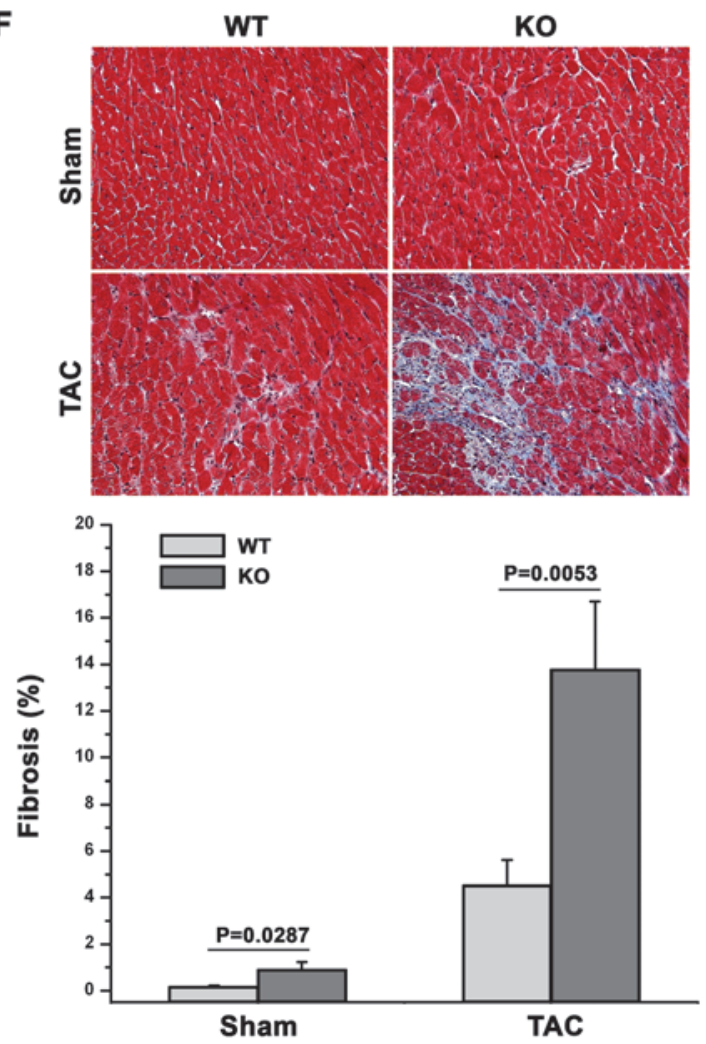


\section{Figure 3}

TAC in 2-month-old KO mice. Mice underwent echocardiography and then were subjected to TAC or sham TAC. Mice were followed with weekly echocardiography, until sacrifice at 3 weeks after TAC. (A) LV mass. Note the exaggerated hypertrophic response in the KO mice. Black triangles represent WT mice subjected to TAC; red circles represent KO mice subjected to TAC; and green and blue squares represent WT and KO shams, respectively. (B) Fractional shortening in the KO mice progressively declined over the 3 weeks after TAC, whereas fractional shortening in the WT mice remained stable. (C) EDD progressively declined in WT and KO mice for the first 2 weeks but then increased sharply in the $\mathrm{KO}$ mice, consistent with adverse remodeling/dilatation. (A-C) $P$ values are for the comparison between WT versus KO mice subjected to TAC. $n=8 \mathrm{WT}$ and $8 \mathrm{KO}$ sham mice; $n=20 \mathrm{WT} / \mathrm{TAC}$ and $20 \mathrm{KO} / \mathrm{TAC}$. (D) LVSP, measured by invasive hemodynamic evaluation, was decreased in the KO mice subjected to TAC. $n=8$ WT and 8 KO sham mice; $n=11 \mathrm{WT} / \mathrm{TAC}$ and $9 \mathrm{KO} / \mathrm{TAC}$. (E) Morphometrics (hypertrophy). $\mathrm{HW} / \mathrm{BW}$ was determined at sacrifice in KO and WT mice undergoing TAC or sham surgery. $n=8 \mathrm{WT}$ and $8 \mathrm{KO}$ sham mice; $n=9 \mathrm{WT} / \mathrm{TAC}$ and $11 \mathrm{KO} / \mathrm{TAC}$. (F) Fibrosis in the $\mathrm{KO}$ mice. WT and $\mathrm{KO}$ mice that underwent TAC were sacrificed at 3 weeks. Sections of heart tissue were stained with Masson's trichrome. The percentage of fibrosis was determined from 10 random images taken from each of $4 \mathrm{KO}$ and 4 WT mice. Original magnification, $\times 200$.

examined cAMP production in response to isoproterenol infusion in the $\mathrm{KO}$ mouse and found that it was markedly reduced compared with that of the WT mouse, both in the heart in vivo (Figure $8 \mathrm{~A}$ ) and in cardiomyocytes isolated from the $\mathrm{KO}$ mouse (Figure $8 \mathrm{~B}$ ). To confirm that the dysregulation of cAMP production in the KO mouse was due to a direct effect of deletion of Gsk3a, we used SB415286, a relatively selective, small molecule inhibitor of GSK-3 $(25,26)$. Pretreatment of WT cardiomyocytes for 30 minutes with SB415286 led to a significant reduction in the contractile response to isoproterenol, mimicking the findings in the KO cells (Figure 8C). Furthermore, SB415286 significantly blunted isoproterenol-induced cAMP production in neonatal rat ventricular myocytes (NRVMs), although the cells were comparable in their ability to generate cAMP following forskolin (Figure 8D).

We then explored the consequences of the impaired cAMP production on downstream signaling. We first examined a central target of PKA, phospholamban (PLN), in 2-month-old mice. Isoproterenol-induced phosphorylation of PLN at the PKA (and CamKII) sites was markedly reduced in the KO mice (Figure 8E). These alterations in $\beta$-adrenergic-mediated PLN phosphorylation can be expected to lead to inhibition of SERCA2 and, if sustained, to impaired calcium handling and, eventually, contractile dysfunction.

Based on our studies, we knew that the defect in cAMP production was upstream of adenylyl cyclase and therefore was likely very proximal in the signaling cascade. A parallel signaling pathway to the adenylyl cyclase/cAMP/PKA pathway that is typically activated when the adenylyl cyclase/cAMP response is downregulated by $\beta$-arrestin involves $\beta$-arrestin-mediated internalization of receptor and activation of ERK1/2 $(27,28)$. Although ERK1/2 was only minimally activated in the basal state in the KO hearts, isoproterenol-induced ERK1/2 activation was significantly increased in the KO mice (Figure 8F).

Our studies, taken together, demonstrate that $\beta$-adrenergic responsiveness is directly regulated by GSK- $3 \alpha$, and GSK- $3 \alpha$-medi- ated positive regulation of $\beta$-adrenergic responsiveness is essential to the heart's adaptation to stress. In the absence of GSK-3 $\alpha$, the stressed $L V$ rapidly remodels and fails.

\section{Discussion}

In this manuscript, we identify GSK-3 $\alpha$ as a critical regulator of hypertrophic growth of the nonstressed heart, of the hypertrophic response to pressure load, and of maintenance of contractile function, particularly in the setting of stress. In the absence of GSK- $3 \alpha$, a spontaneous cardiomyopathy develops that is characterized by relatively marked hypertrophy and modest LV dysfunction, with striking mitochondrial structural abnormalities later in life. Most importantly, we find that GSK-3 $\alpha$ is a critical and direct regulator of $\beta$-adrenergic responsiveness. In the absence of GSK- $3 \alpha$, the LV is unable to adapt to pressure stress, leading to a rapid deterioration in function and to decompensation, including a striking inability to sustain a pressor response. This culminates in adverse remodeling with LV dilatation. How does deletion of Gsk3a lead to these phenotypes?

GSK-3 $\alpha$ regulates bypertrophy. The role of GSK- $3 \beta$ in cardiac hypertrophy has been examined extensively by several groups, and the vast majority of studies have found that expression of either WT or a constitutively active S9A mutant repressed hypertrophy (8-14). However, based on studies with overexpression of a dominant inhibitory mutant of GSK-3 $\beta$, which showed no enhancement of hypertrophy following TAC, it was not clear what role, if any, inhibition of GSK-3 $\beta$ played in the response (12). Findings with GSK-3 $\alpha$ have been even less clear. Whereas a constitutively active transgenic repressed the hypertrophic response to TAC, the same mutant expressed in a knockin model showed an increased hypertrophic response $(13,14)$. These disparate and confusing findings, which led Sugden et al. to state "we just do not know whether to inhibit or activate GSK-3, or simply not to interfere" (29), highlight the need to examine loss-of-function approaches that do not rely on transgenesis. Our data clearly identify a key role for GSK-3 $\alpha$ as a negative regulator of hypertrophic growth, whether it be in response to stress or not.

In screening for mechanisms by which GSK-3 $\alpha$ regulates hypertrophy, we identified mTORC1 as a key target of the kinase, and loss of GSK- $3 \alpha$ cannot be compensated for by GSK- $3 \beta$, in regards to this pathway. This is surprising, since the few studies done to date have focused on GSK-3 $\beta$ and its role in regulating mTORC1 downstream of Wnt signaling $(30,31)$. Our studies identify GSK-3 $\alpha$ as a central regulator of $\mathrm{mTORC} 1$ in hypertrophic signaling. In the absence of GSK-3 $\alpha$, activity of mTORC1 is significantly increased. Given the critical role played by $\mathrm{mTORC} 1$ in regulating the protein translation machinery (30), this is very likely to be a key mechanism by which GSK-3 $\alpha$ regulates the hypertrophic response. In addition to dysregulation of mTORC1, it is likely that the contractile dysfunction seen, especially later in the life of the KO mouse, plays a role in the increased hypertrophy as a maladaptive response.

$G S K-3 \alpha$ regulates contractile function. Prior studies examining effects of GSK-3s on TAC-induced LV dysfunction have suggested that increased activity of GSK-3 $\alpha$, by either transgenesis or knockin, led to declines in LV function, suggesting GSK-3 $\alpha$ signaling is detrimental $(13,14)$. In distinct contrast, our lossof-function studies identify a clear and critical role for GSK-3 $\alpha$ in preserving LV function in the setting of pressure stress. In the presence of GSK-3 $\alpha$, hearts developed compensated hypertrophy in response to TAC, whereas in the absence of GSK-3 $\alpha$, 
A

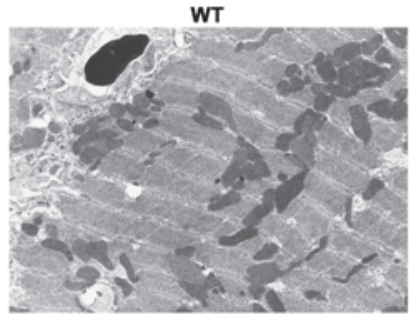

C

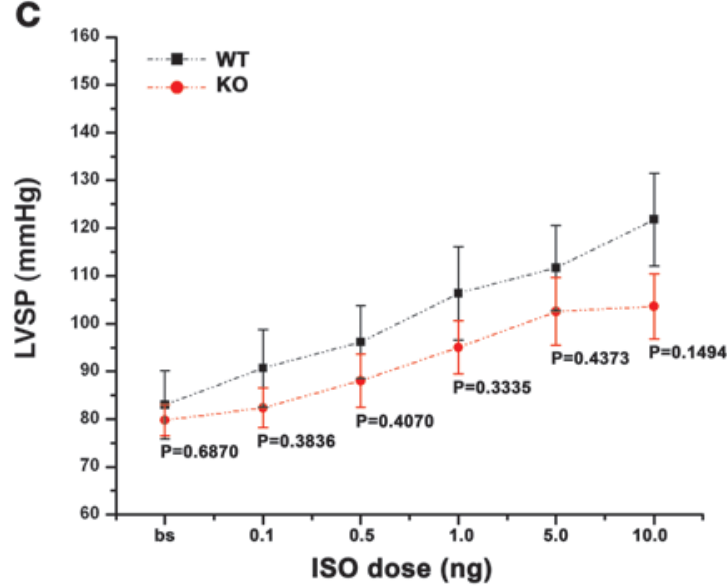

E

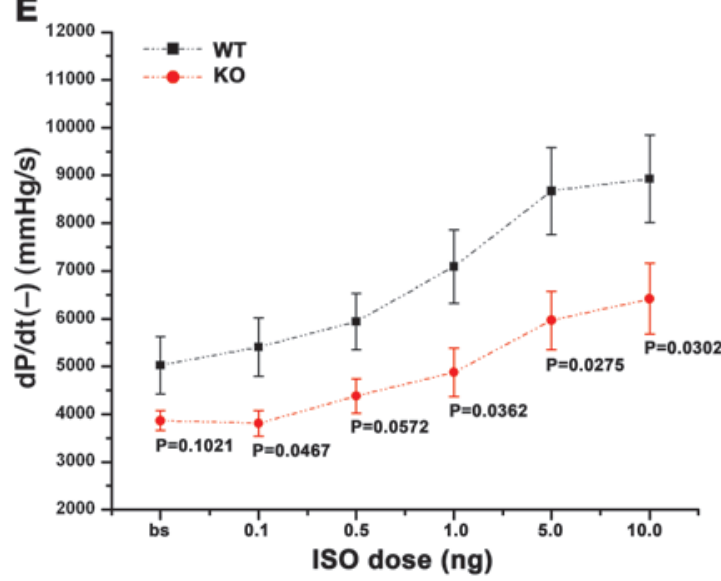

B

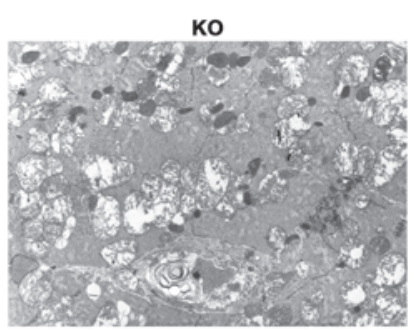

D

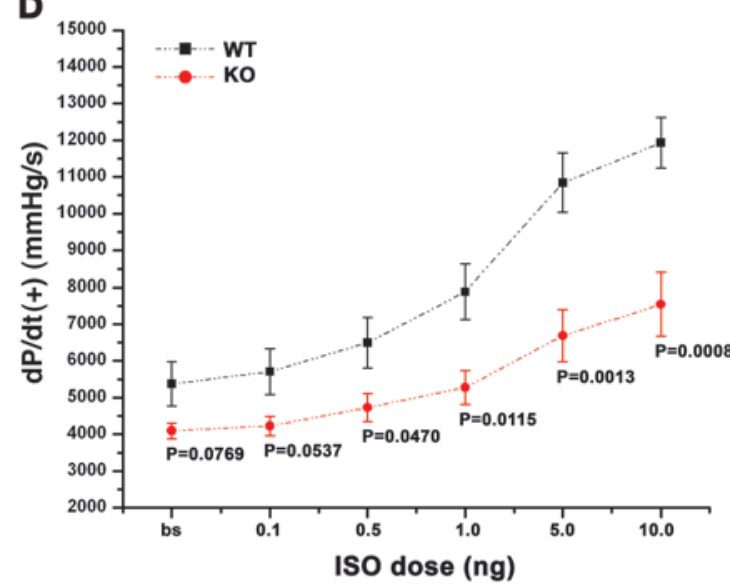

F

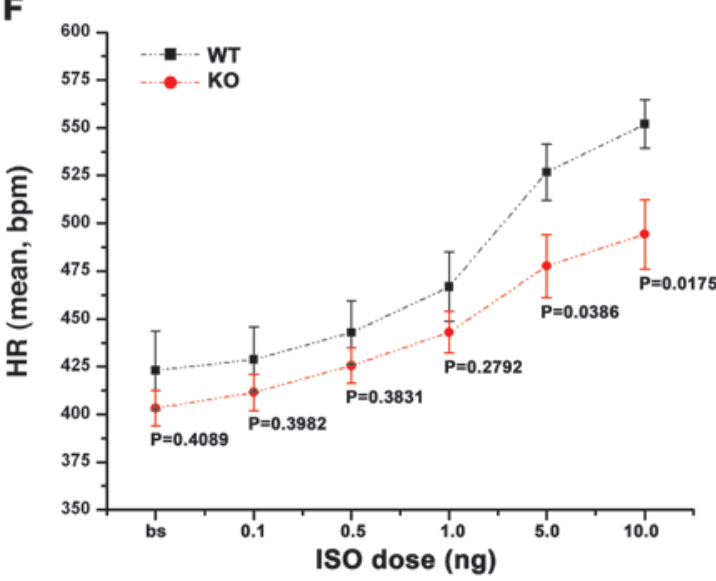

Figure 4

Ultrastructural abnormalities and impaired $\beta$-adrenergic responsiveness in the KO mouse. $(\mathbf{A}$ and $\mathbf{B})$ Mitochondrial structural abnormalities in the KO mouse. Six-month-old WT (A) and KO (B) mouse hearts were fixed and processed for transmission EM. In the KO, images show marked swelling of many mitochondria, with only remnants of cristae remaining. For comparison, several normal mitochondria are scattered throughout the section and are also evident in A. Original magnification, $\times 5,000$. (C-F) Impaired response to graded isoproterenol (ISO) infusion in 6-monthold KO versus WT mice. Mice were subjected to graded isoproterenol infusion, as described in Methods. LVSP (C), +dP/dt (D), $-\mathrm{dP} / \mathrm{dt}(\mathbf{E})$, and heart rate $(\mathrm{HR})(\mathrm{F})$ responses are shown. $P$ values, comparing KO to WT mice, are displayed for each of the doses of isoproterenol. $n=8$ WT and $13 \mathrm{KO}$ mice. bs, basal.

the hearts undergo striking and accelerated adverse remodeling, with decompensation. While the marked disruption of mitochondrial structural integrity may have played a role in the LV dysfunction, given the late appearance of this finding (at 6 months), this is clearly not the primary defect. Rather, we believe that our studies identify the critical mechanism underlying the protective effects of GSK-3 $\alpha$ in the stressed heart to be maintenance of $\beta$-adrenergic responsiveness. In the absence of GSK- $3 \alpha$, there is marked dysregulation of $\beta$-adrenergic signaling that results in markedly reduced phosphorylation of PLN.
The altered $\beta$-adrenergic signaling is apparent prior to the mitochondrial abnormalities and prior to hypertrophy, suggesting it is not a secondary response. This is supported by the findings with the forskolin derivative, NKH477 (in response to which the $\mathrm{KO}$ mouse had a comparable increase in contractile performance to that of the WT mouse), and with the small molecule inhibitor that acutely recapitulated the altered $\beta$-adrenergic responsiveness in WT mouse cardiomyocytes and in NRVMs.

Our studies indicate that GSK- $3 \alpha$ interacts with the $\beta$-adrenergic system above the level of PKA, since cAMP production is 

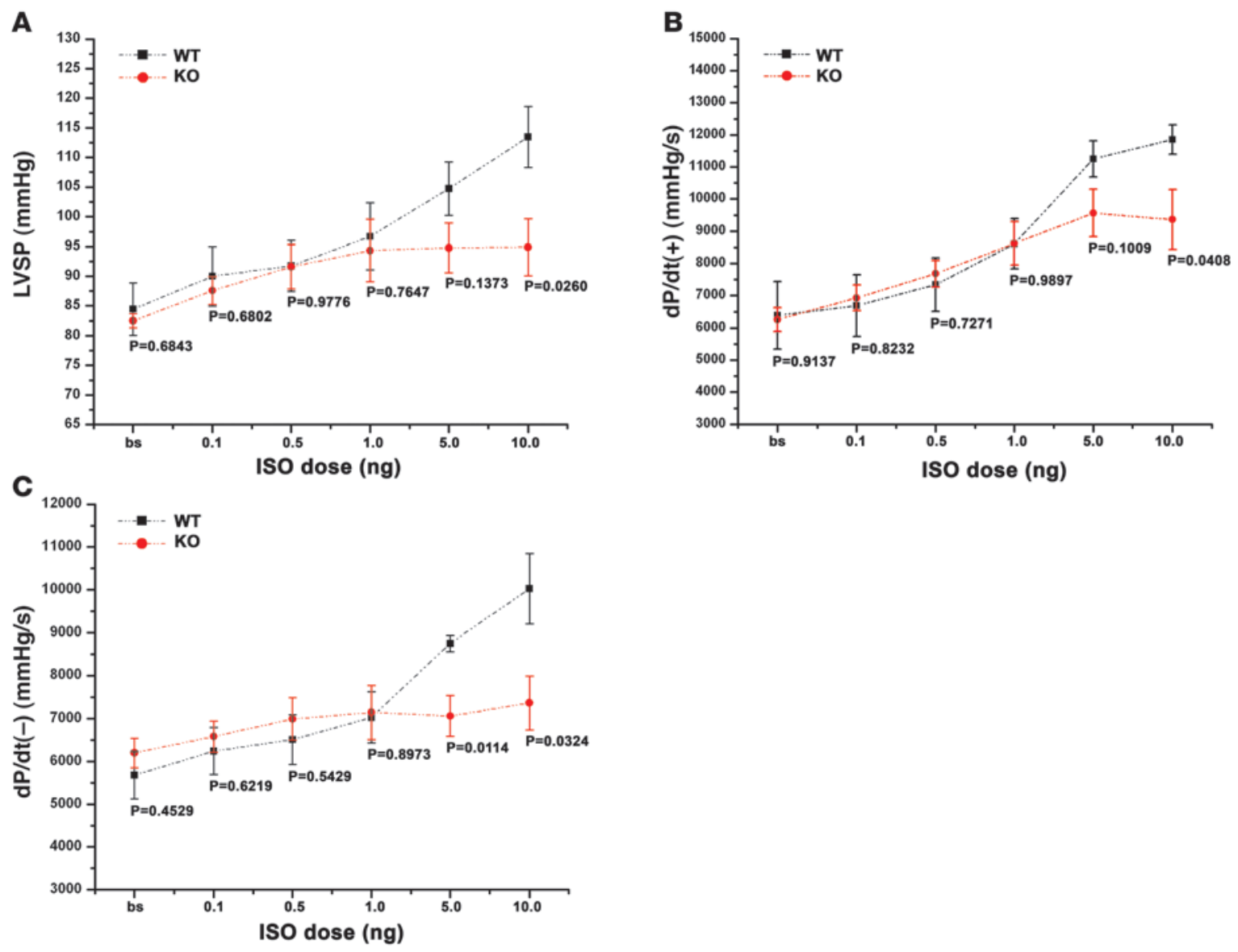

Figure 5

Impaired response to graded isoproterenol infusion in 2-month-old KO versus WT mice. LVSP $(\mathbf{A}),+d P / d t(B)$, and -dP/dt $(\mathbf{C})$ were all reduced in the KO mice. $P$ values, comparing KO to WT mice, are displayed for each of the doses of isoproterenol. $n=5 \mathrm{WT}$ and $7 \mathrm{KO}$ mice.

reduced in the $\mathrm{KO}$ mice, and above the level of adenylyl cyclase, since forskolin-induced cAMP production was not reduced by treatment of WT cardiomyocytes with the GSK-3 inhibitor (Figure 8D). Thus the site of interaction is very proximal, presumably at the receptor/G protein-coupled receptor kinase/ $\beta$-arrestin level. The decreased $\beta$-adrenergic responsiveness, taken together with enhanced ERK1/ 2 activation in the KO mouse, suggests increased $\beta$-arrestin activity is involved, since $\beta$-arrestin leads to both downregulation of $\beta$-adrenergic signaling through $\mathrm{G}_{\mathrm{s}} /$ adenylyl cyclase/cAMP and increased ERK1/2 signaling via increased internalization of receptors and ERKs.

The physiological role of this pathway seems clear: GSK-3 $\alpha$ normally serves as a positive feedback loop to enhance $\beta$-adrenergic signaling. Given that PKA can phosphorylate GSK-3 $\alpha$, thereby inhibiting the kinase $(32,33)$, this would provide a mechanism for limiting $\beta$-adrenergic signaling. Obviously, however, if the inhibition is marked and sustained, serious adverse consequences can result.

Potential clinical implications and conclusions. Our data underline the critical role that GSK-3 $\alpha$ plays in the heart's response to stress. This is a significant concern, given the proposed use of inhibitors of GSK-3 as insulin sensitizers and as agents to treat bipolar disorders and a host of other disease states, including neurodegenerative disorders and ischemic injury $(2,18,19,25$,
$34,35)$. On the other hand, in patients with preserved LV function, moderate inhibition of GSK-3 $\alpha$ could potentially be used as a novel strategy to achieve $\beta$-blockade, without leading to the well-described $\beta$-blocker-induced deteriorations in glucose control (36). However, given our findings in the $\mathrm{KO}$ mouse, the toxic-to-therapeutic ratio of any such inhibitor would be a major concern.

In summary, we believe we have identified a novel and central role for GSK-3 $\alpha$ in regulating contractile function, particularly in the setting of stress, and this is mediated via direct regulation of the $\beta$-adrenergic signaling pathway.

\section{Methods}

Antibodies. Antibodies used were directed against GSK-3 $\alpha$ (catalog 9338), GSK-3 $\beta$ (catalog 9315), glycogen synthase (catalog 3893), glycogen synthase phosphorylated at Ser641 (catalog 3891), elongation factor 4Ebinding protein 1 (4E-BP1) (catalog 9644), 4E-BP1 phosphorylated at Thr37/46 (catalog 9459), 4E-BP1 phosphorylated at Ser65 (catalog 9451), and p70S6K phosphorylated at Thr389 (catalog 9206) (all from Cell Signaling Technology). p70S6K (catalog 611260) was from BD Biosciences - Transduction Laboratories. PLN, clone 1 (catalog 05-205) was from Millipore. PLN phosphorylated at Ser16 (catalog A010-12) or at Thr17 (catalog A010-13) were from Badrilla Ltd. GAPDH (catalog RDITRK5D4-6C5) was from Research Diagnostics. 

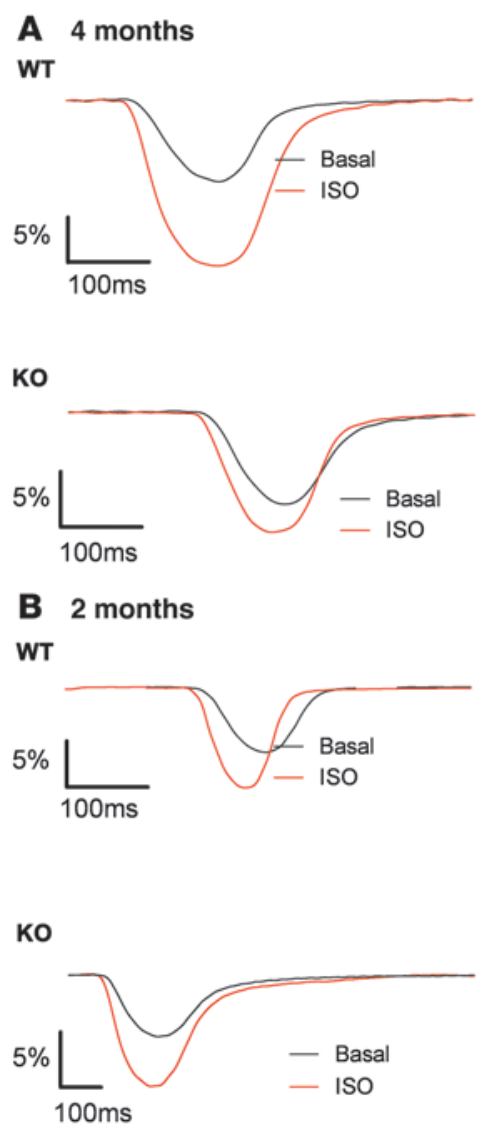
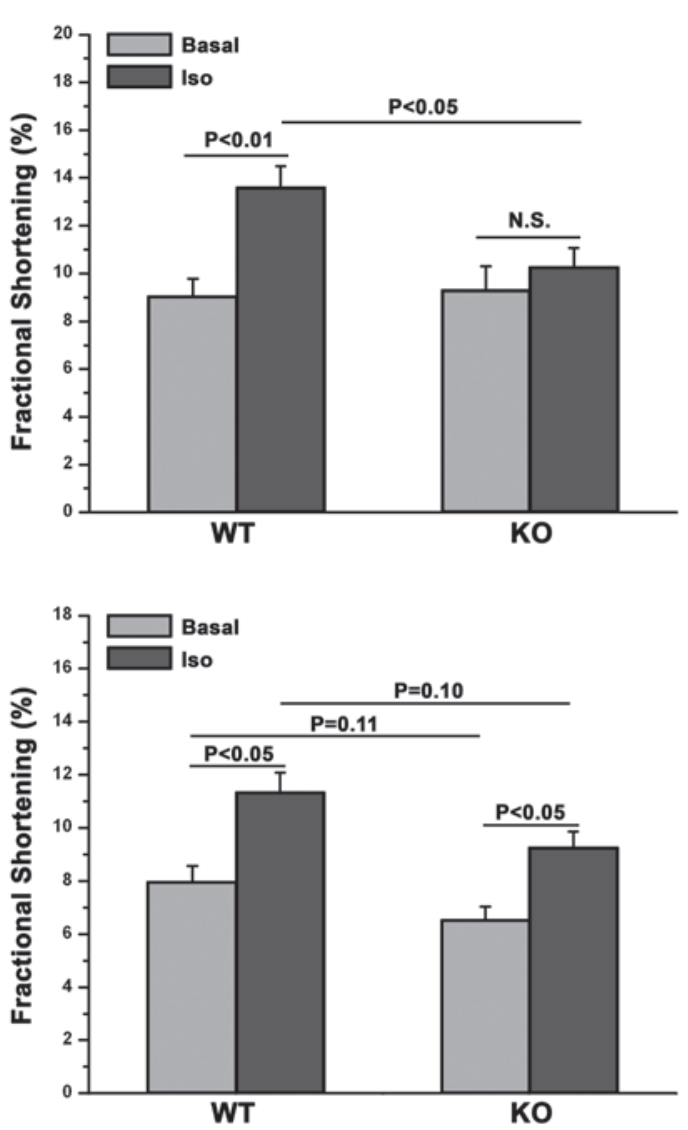

\section{Figure 6}

Contractile function of cardiomyocytes isolated from WT and KO mice. (A and B) Cardiomyocytes were isolated from 4-month-old (A) and 2-month-old (B) WT and $\mathrm{KO}$ mice, as described in Methods. Basal and isoproterenol-stimulated fractional shortening were determined at a $5 \times 10^{-7} \mathrm{M}$ concentration of isoproterenol. Representative tracings from WT and KO cells and quantification are shown graphically. (A) Quantification is derived from 14 WT and $14 \mathrm{KO}$ cells derived from mice $(n=6)$. (B) Quantification is from 23 WT and $23 \mathrm{KO}$ cells derived from mice $(n=6)$.
Chemicals. The GSK-3 inhibitor, SB415286 (catalog S3567), the adenylyl cyclase activator, forskolin (catalog F6886), and the protease and phosphatase inhibitor cocktails (catalog P8430, P2850) were from SigmaAldrich. Isoproterenol was from Hospira (catalog 0409-1410-05).

Immunoblotting. $\mathrm{LV}$ tissue was homogenized in 10 volumes of lysis buffer (50 mM Tris-HCl [pH 7.4]), $150 \mathrm{mM} \mathrm{NaCl}, 1 \mathrm{mM}$ EDTA, $0.25 \%$ sodium deoxycholate, $1 \% \mathrm{NP}-40$, with the protease inhibitor cocktail and phosphatase inhibitor cocktail present. After homogenization, the homogenates were centrifuged at $15,000 \mathrm{~g}$ for 15 minutes and separated into NP-40-soluble supernatant and insoluble pellet. Protein concentration in the supernatant was quantified with the bicinchoninic acid protein assay (catalog 23225; Pierce). Equal amounts of proteins were subjected to SDS-PAGE and subsequently were transferred to nitrocellulose membranes. Primary antibody incubations were performed at 1:1,000 dilution. All incubations were done at $4{ }^{\circ} \mathrm{C}$, overnight. The secondary antibody used was Alexa Fluor 680 (Molecular Probes), at 1:3,000 dilution for 1 hour at room temperature. Membranes were scanned with the Odyssey Infrared Imaging System (LI-COR).

Myocyte isolation and determination of cardiomyocyte surface area. Mouse LV myocytes were isolated as previously described $(37,38)$. The percentage of rod-shaped myocytes was greater than $70 \%$. Isolated cardiomyocytes were fixed in $0.1 \mathrm{M}$ PBS containing $2 \%$ paraformaldehyde and $2.5 \%$ glutaraldehyde. A $30 \mu \mathrm{l}$ suspension of isolated myocytes were loaded onto positively charged slides and air dried. Myocytes were then placed in $0.1 \mathrm{M}$ glycine in PBS for 30 minutes and then were washed with PBS. Cells were permeabilized in $1 \%$ Triton X-100 in PBS ( $\mathrm{pH} 7.2$ ) at room temperature for 20 minutes. Cells were then washed in PBS and incubated in 10\% Wheat Germ Agglutinin (catalog F49; Biomeda) in PBS with 2\% BSA for 1 hour.
Slides were washed 3 times with PBS, for 10 minutes each. Slides were then coated with Immo-Mount Mounting Medium for fluorescence (catalog H-1000; Vector). The surface area of cardiomyocytes was then quantified using NIS-Elements Imaging Software (Nikon) (39).

Contractile function in isolated cardiomyocytes. Ventricular myocytes were isolated as described above. Myocytes were maintained at room temperature in $5 \% \mathrm{CO}_{2}$ and $95 \% \mathrm{O}_{2}$ and were used within 8 hours of isolation. Myocytes were placed in a chamber mounted on an inverted Nikon microscope and perfused with Tyrode's solution containing $1 \mathrm{mM} \mathrm{Ca}^{2+}$. Cells were paced at $1 \mathrm{~Hz}$ and contractions were measured with a video edge detector as described previously $(37,38,40)$.

cAMP assay. For determination of cAMP content, we used the EIA Kit for Cell and Tissue Lysates from BIOMOL (catalog AK-205). Briefly, to determine the level of cAMP in the heart, $\mathrm{LV}$ tissue was homogenized in 10 volumes of $0.1 \mathrm{M}$ hydrochloric acid. For determination of cAMP content in isolated mouse LV myocytes and NRVMs, $10^{6}$ cells were sonicated in $300 \mu \mathrm{l}$ of $0.1 \mathrm{M}$ hydrochloric acid. Thereafter, the procedure followed the manufacturer's instructions.

Echocardiographic and hemodynamic analysis of cardiac function. Transthoracic 2-dimensional echocardiography (TTE) was performed in anesthetized ( $2 \%$ inhaled isoflurane) WT and Gsk3a-/- $(\mathrm{KO})$ mice with a $12-\mathrm{MHz}$ probe $(39,41)$. TTE in M-mode was carried out in the parasternal short-axis view to assess $\mathrm{LV}$ end-diastolic and end-systolic diameters and function (assessed as fractional shortening).

For in vivo hemodynamic measurements, a 1.4 French micromanometer-tipped catheter (SPR-671; Millar Instruments Inc.) was inserted into the right carotid artery and advanced into the $\mathrm{LV}$ of mice that were lightly anesthetized (i.e., maintained spontaneous respirations) 

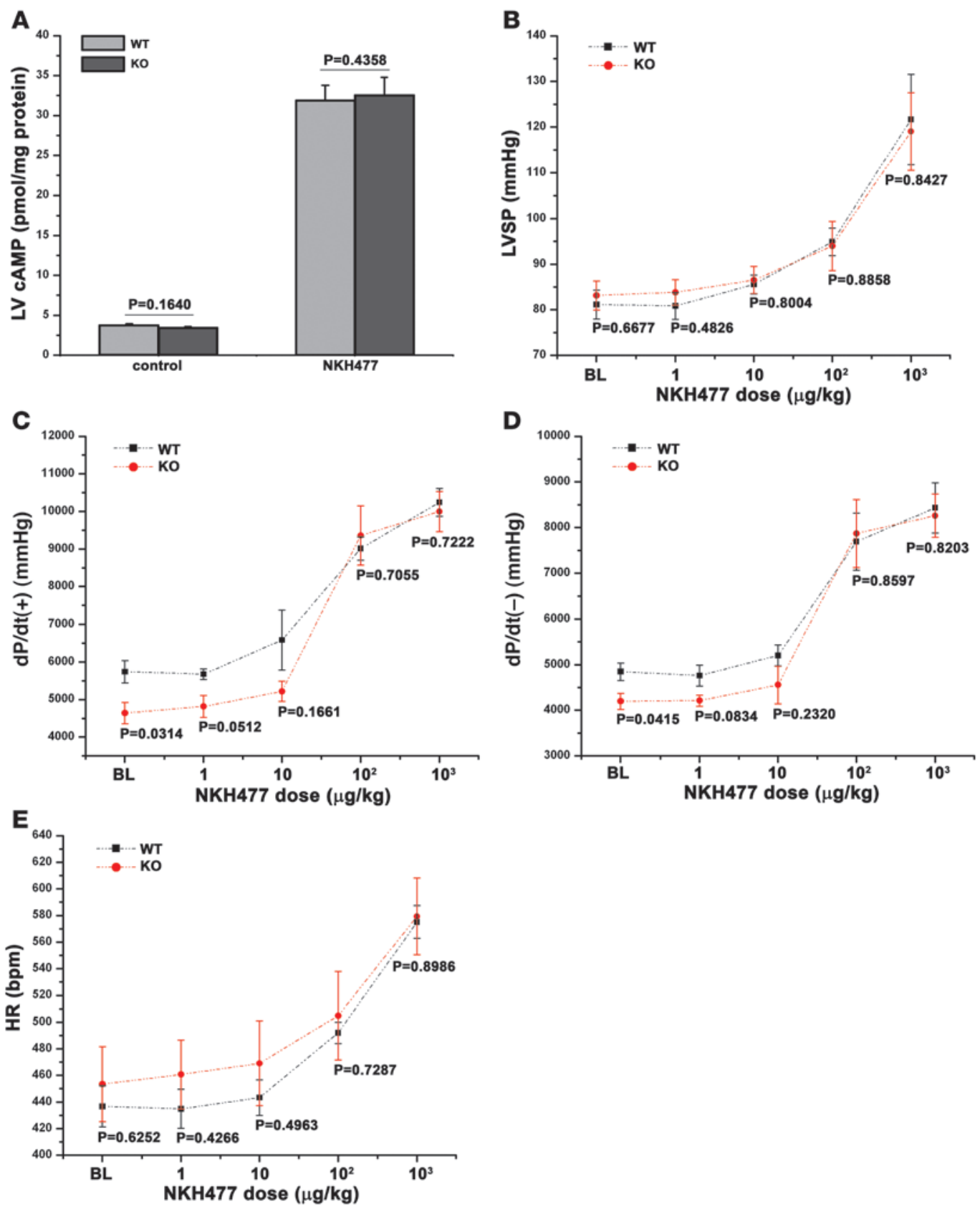

Figure 7

Normal contractile function in the KO mice following direct activation of adenylyl cyclase. (A) cAMP production in 4-month-old WT and KO hearts, following graded NKH477 infusion, as described in Methods. $n=5 \mathrm{WT}$ and $5 \mathrm{KO}$ control hearts; $n=7 \mathrm{WT}$ and $6 \mathrm{KO}$ hearts treated with NKH477. (B-E) Hemodynamic response to graded NKH477 infusion. LVSP (B), +dP/dt (C), -dP/dt (D), and heart rate (E). BL, baseline measurement prior to administration of NKH477 versus vehicle. $n=9$ WT and $7 \mathrm{KO}$ hearts.

with tribromoethanol/amylene hydrate $(2.5 \% \mathrm{wt} / \mathrm{vol}, 8 \mu \mathrm{l} / \mathrm{g}$ injected intraperitoneal; Avertin). Hemodynamic parameters, including heart rate, $\mathrm{LV}$ end-diastolic pressure, and $\mathrm{dP} / \mathrm{dt}$ and $-\mathrm{dP} / \mathrm{dt}$, were recorded in closed-chest mode, both at baseline and in response to increasing doses of isoproterenol $(0.1,0.5,1,5$, and $10 \mathrm{ng})$, administered via cannulation of the right internal jugular vein (42).

For the studies with NKH477 (catalog 1063; TOCRIS), mice were anesthetized and instrumented as above. NKH477 was dissolved in PBS and was administered in doses of $1,10,100$, and $1,000 \mu \mathrm{g} / \mathrm{kg}$ in
5 -minute intervals, thereby allowing peak contractile function to be reached prior to dose escalation. Hemodynamic parameters were exactly as described above.

TAC. TAC was performed as previously described (39). Briefly, mice were sedated with isoflurane (induction, $3 \%$; maintenance, $1.5 \%$ ) and anesthetized to a surgical plane with intraperitoneal ketamine $(50 \mathrm{mg} / \mathrm{kg})$ and xylazine $(2.5 \mathrm{mg} / \mathrm{kg})$. Anesthetized mice were intubated, and a midline cervical incision was made to expose the trachea and carotid arteries. A blunt 20-gauge needle was inserted into the trachea and con- 
A

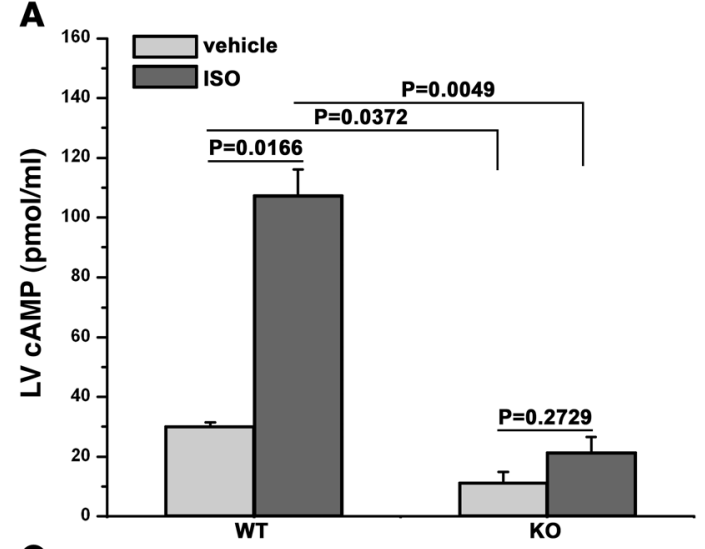

C

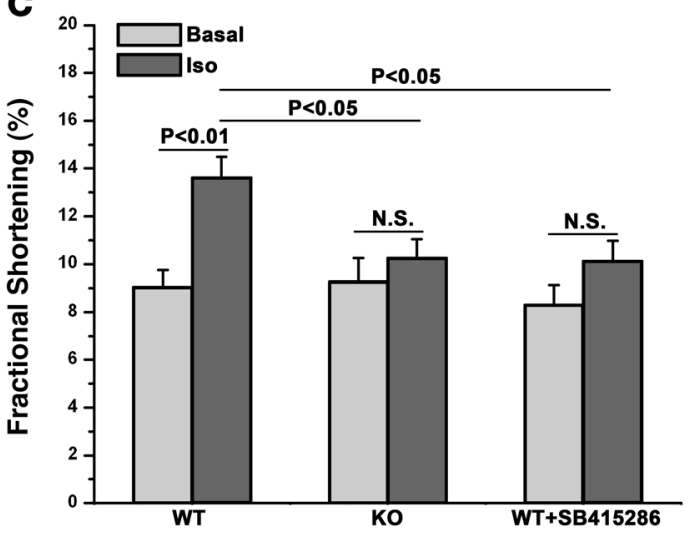

$\mathbf{E}$

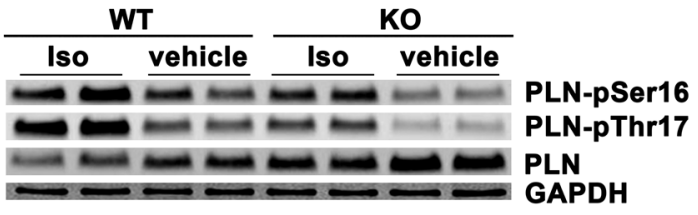

B

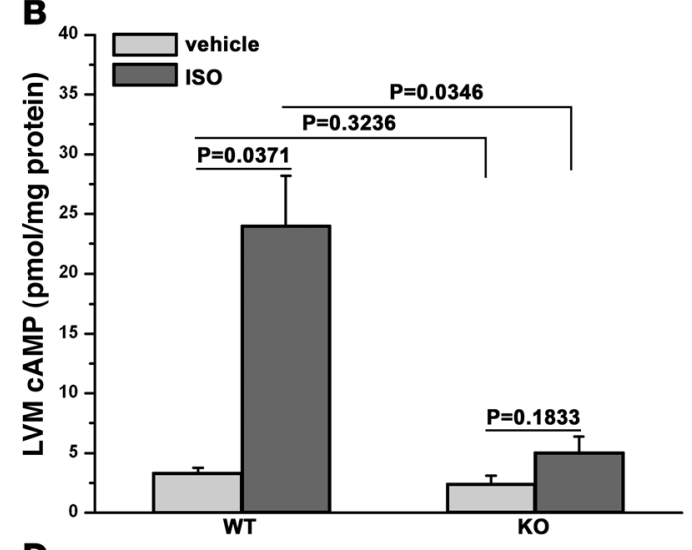

D

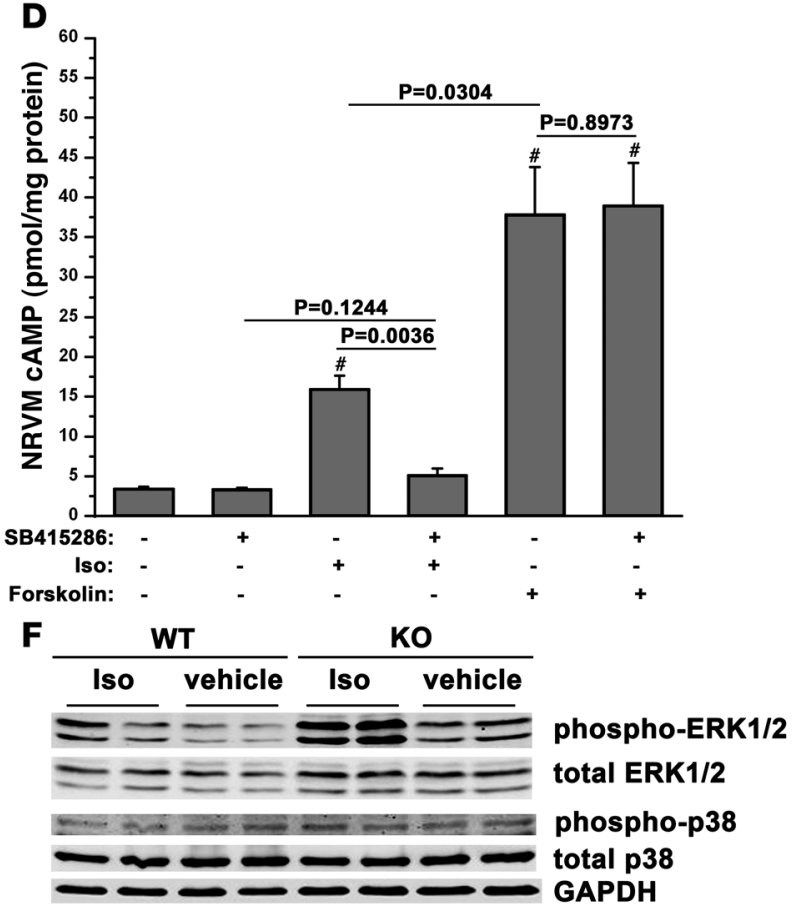

Figure 8

GSK-3 $\alpha$ regulates isoproterenol-induced cAMP production. (A-C) Studies were in 4-month-old mice. (A) cAMP production in the intact mouse heart. KO and WT mice were injected with isoproterenol (1 ng/g BW) versus vehicle. Fifteen minutes later, hearts were excised, and cAMP production was determined. $n=4$ mice per condition. (B) cAMP production in response to vehicle versus isoproterenol $(5 \mu \mathrm{M}, 15$ minutes) in cardiomyocytes isolated from KO and WT mice $(n=6)$, with $4 \times 10^{5}$ myocytes per assay. LVM, LV myocyte. (C) Inhibition of isoproterenol-induced contractile function by a GSK-3 inhibitor. Cardiomyocytes were isolated from WT and KO mice $(n=6)$. Fractional shortening was determined at baseline, after isoproterenol (5 $\mu \mathrm{M}, 10$ minutes), and in WT cells pretreated with SB415286 (10 $\mu \mathrm{M}, 60$ minutes). Contractile function increased with isoproterenol in WT cells but not in KO cells or SB415286-treated WT cells. $n=14$ cells for WT and KO mice; $n=12$ cells for WT plus SB415286 mice. (D) SB415286 blocks cAMP production in NRVMs. NRVMs were pretreated with SB415286 or vehicle, followed by isoproterenol versus vehicle. SB415286 reduced isoproterenol-induced cAMP production but not forskolin-induced cAMP production. (E) Reduced isoproterenolinduced phosphorylation of PLN in the KO mice. Two-month-old WT and KO mice underwent isoproterenol infusion (1 $\mu \mathrm{g} / \mathrm{kg} \mathrm{BW})$ for $15 \mathrm{minutes}$. Lysates were immunoblotted for PLN phosphorylated at Ser16 (PKA site), Thr17 (CamKII site), total PLN, and GAPDH. (F) Increased isoproterenol-induced ERK1/2 activation in the KO mice. Mice were treated with isoproterenol versus vehicle as above, and cell lysates were immunoblotted for phospho-ERK1/2, total ERK1/2, phospho-p38, total p38, or GAPDH.

nected to a volume-cycled rodent ventilator on supplemental oxygen at a rate of $1 \mathrm{l} / \mathrm{min}$, with a respiratory rate of $140 \mathrm{breaths} / \mathrm{min}$. Aortic constriction was performed by tying a 7-0 nylon suture ligature against a 27 -gauge needle. The needle was then promptly removed to yield a constriction of approximately $0.4 \mathrm{~mm}$ in diameter. LVSP was determined by in vivo hemodynamics, with a high fidelity pressure transducer advanced to the LV.
Transmission electron microscopy. Transmission electron microscopy was performed as previously described $(41,43)$.

Statistics. Comparisons between 2 groups were performed using 2-tailed Student's $t$ test. Comparisons between multiple groups were performed using 1-way ANOVA with Holm-Sidak post-test using SigmaStat software (Systat). All data are presented as mean \pm SEM, except the tables, which are mean \pm SD. A $P$ value of less than 0.05 was considered significant. 
All procedures herein were approved by the Institutional Animal Care and Use Committee of Thomas Jefferson University.

\section{Acknowledgments}

This work was supported by NIH grants HL061688 and HL091799 (to T. Force), by the Canadian Institutes of Health Research grants MOP 12858 and 74711 (to J. Woodgett), and by donations from The Kahn Foundation and the Scarperi family.

1. Doble BW, Woodgett JR. GSK-3: tricks of the trade for a multi-tasking kinase. J Cell Sci. 2003 116(pt 7):1175-1186.

2. Rayasam GV, Tulasi VK, Sodhi R, Davis JA, Ray A. Glycogen synthase kinase 3: more than a namesake. Br J Pharmacol. 2009;156(6):885-898.

3. Force T, Woodgett JR. Unique and overlapping functions of GSK-3 isoforms in cell differentiation and proliferation and cardiovascular development. J Biol Chem. 2009;284(15):9643-9647.

4. Ruel L, Bourouis M, Heitzler P, Pantesco V, Simpson P. Drosophila shaggy kinase and rat glycogen synthase kinase- 3 have conserved activities and act downstream of Notch. Nature. 1993;362(6420):557-560.

5. Siegel JN, Klausner RD, Rapp UR, Samelson LE. $T$ cell antigen receptor engagement stimulates $\mathrm{c}$ raf phosphorylation and induces c-raf associated kinase activity via a protein kinase C-dependent pathway. J Biol Chem. 1990;265(30):18472-18480.

6. Doble BW, Patel S, Wood GA, Kockeritz LK, Woodgett JR. Functional redundancy of GSK-3alpha and GSK-3beta in Wnt/beta-catenin signaling shown by using an allelic series of embryonic stem cell lines. Dev Cell. 2007;12(6):957-971.

7. Kerkela R, et al. Deletion of GSK-3beta in mice leads to hypertrophic cardiomyopathy secondary to cardiomyoblast hyperproliferation. J Clin Invest. 2008;118(11):3609-3618.

8 . Antos CL, et al. Activated glycogen synthase kinase- $3 \beta$ suppresses cardiac hypertrophy in vivo. Proc Natl Acad Sci U S A. 2002;99(2):907-912.

9. Michael A, et al. Glycogen synthase kinase- $3 \beta$ regulates growth, calcium homeostasis, and diastolic function in the heart. $J$ Biol Chem. 2004;279(20):21383-21393

10. Sanbe A, Gulick J, Hanks MC, Liang Q, Osinska $\mathrm{H}$, Robbins J. Reengineering inducible cardiac-specific transgenesis with an attenuated myosin heavy chain promoter. Circ Res. 2003;92(6):609-616.

11. Trivedi CM, et al. $\mathrm{Hdac} 2$ regulates the cardiac hypertrophic response by modulating Gsk3beta activity. Nat Med. 2007;13(3):324-331.

12. Hirotani $S$, et al. Inhibition of glycogen synthase kinase 3 beta during heart failure is protective. Circ Res. 2007;101(11):1164-1174.

13. Matsuda T, et al. Distinct roles of GSK-3alpha and GSK-3beta phosphorylation in the heart under pressure overload. Proc Natl Acad Sci U S A. 2008;105(52):20900-20905.

14. Zhai P, et al. Glycogen synthase kinase-3alpha reduces cardiac growth and pressure overloadinduced cardiac hypertrophy by inhibition of extracellular signal-regulated kinases. J Biol Chem.

Received for publication October 12, 2009, and accepted in revised form April 7, 2010.

Address correspondence to: Thomas Force, Center for Translational Medicine, Cardiology Division, Thomas Jefferson University, College Building, Rm. 316, 1025 Walnut St., Philadelphia, Pennsylvania 19107, USA. Phone: 215.503.9520; Fax: 215.503.5731; E-mail: thomas.force@jefferson.edu.

2007;282(45):33181-33191

15. Bikkavilli RK, Feigin ME, Malbon CC. p38 mitogenactivated protein kinase regulates canonical Wntbeta-catenin signaling by inactivation of GSK3beta. J Cell Sci. 2008;121(pt 21):3598-3607.

16. Thornton TM, et al. Phosphorylation by p38 MAPK as an alternative pathway for GSK3beta inactivation. Science. 2008;320(5876):667-670.

17. McManus EJ, et al. Role that phosphorylation of GSK3 plays in insulin and Wnt signaling defined by knockin analysis. EMBOJ. 2005;24(8):1571-1583.

18. Patel S, Doble BW, MacAulay K, Sinclair EM, Drucker DJ, Woodgett JR. Tissue-specific role of glycogen synthase kinase 3beta in glucose homeostasis and insulin action. Mol Cell Biol. 2008;28(20):6314-6328.

19. Tanabe K, et al. Genetic deficiency of glycogen synthase kinase-3beta corrects diabetes in mouse models of insulin resistance. PLoS Biol. 2008;6(2):e37.

20. MacAulay K, et al. Glycogen synthase kinase 3alpha-specific regulation of murine hepatic glycogen metabolism. Cell Metab. 2007;6(4):329-337.

21. Nagy A. Cre recombinase: the universal reagent for genome tailoring. Genesis. 2000;26(2):99-109.

22. Arad M, et al. Glycogen storage diseases presenting as hypertrophic cardiomyopathy. $N$ Engl J Med. 2005;352(4):362-372.

23. Sarbassov DD, Ali SM, Sabatini DM. Growing roles for the mTOR pathway. Curr Opin Cell Biol. 2005;17(6):596-603.

24. Sanbe A, Takeo S. Effects of NKH477, a water-soluble forskolin derivative, on cardiac function in rats with chronic heart failure after myocardial infarction. J Pharmacol Exp Ther. 1995;274(1):120-126.

25. Das S, Wong R, Rajapakse N, Murphy E, Steenbergen C. Glycogen synthase kinase 3 inhibition slows mitochondrial adenine nucleotide transport and regulates voltage-dependent anion channel phosphorylation. Circ Res. 2008;103(9):983-991.

26. Peineau S, et al. LTP inhibits LTD in the hippocampus via regulation of GSK3beta. Neuron. 2007;53(5):703-717.

27. Patel PA, Tilley DG, Rockman HA. Physiologic and cardiac roles of beta-arrestins. J Mol Cell Cardiol. 2009;46(3):300-308.

28. Patel PA, Tilley DG, Rockman HA. Beta-arrestin-mediated signaling in the heart. Circ J. 2008;72(11):1725-1729.

29. Sugden PH, Fuller SJ, Weiss SC, Clerk A. Glycogen synthase kinase 3 (GSK3) in the heart: a point of integration in hypertrophic signalling and a therapeutic target? A critical analysis. Br J Pharmacol. 2008;153 suppl 1:S137-S153.

30. Huang J, Manning BD. The TSC1-TSC2 complex: a molecular switchboard controlling cell growth. Biochem J. 2008;412(2):179-190.

31. Inoki K, et al. TSC2 integrates Wnt and energy signals via a coordinated phosphorylation by AMPK and GSK3 to regulate cell growth. Cell. 2006;126(5):955-968.

32. Li M, Wang X, Meintzer MK, Laessig T, Birnbaum MJ, Heidenreich KA. Cyclic AMP promotes neuronal survival by phosphorylation of glycogen synthase kinase 3 beta. Mol Cell Biol. 2000;20(24):9356-9363.

33. Taurin S, Hogarth K, Sandbo N, Yau DM, Dulin NO. Gbetagamma-mediated prostacyclin production and cAMP-dependent protein kinase activation by endothelin-1 promotes vascular smooth muscle cell hypertrophy through inhibition of glycogen synthase kinase-3. J Biol Chem. 2007;282(27):19518-19525.

34. Rowe MK, Wiest C, Chuang DM. GSK-3 is a viable potential target for therapeutic intervention in bipolar disorder. Neurosci Biobehav Rev. 2007;31(6):920-931.

35. Murphy E, Steenbergen C. Does inhibition of glycogen synthase kinase protect in mice? Circ Res. 2008;103(3):226-228.

36. Klapholz M. Beta-blocker use for the stages of heart failure. Mayo Clin Proc. 2009;84(8):718-729.

37. Chen X, et al. Ca2+ influx-induced sarcoplasmic reticulum $\mathrm{Ca} 2+$ overload causes mitochondrialdependent apoptosis in ventricular myocytes. Circ Res. 2005;97(10):1009-1017.

38. Chen X, et al. Reduced effects of BAY K 8644 on L-type $\mathrm{Ca} 2+$ current in failing human cardiac myocytes are related to abnormal adrenergic regulation. Am JPhysiol Heart Circ Physiol. 2008;294(5):H2257-H2267.

39. Chen X, et al. The $\beta$-catenin/T-cell factor/Lymphocyte enhancer factor signaling pathway is required for normal and stress-induced cardiac hypertrophy. Mol Cell Biol. 2006;26(12):4462-4473.

40. Jaleel N, et al. Ca2+ influx through T- and L-type $\mathrm{Ca} 2+$ channels have different effects on myocyte contractility and induce unique cardiac phenotypes. Circ Res. 2008;103(10):1109-1119.

41. Kerkela R, et al. Cardiotoxicity of the cancer therapeutic agent imatinib mesylate. Nat Med. 2006;12(8):908-916.

42. Gao E, Boucher M, Chuprun JK, Zhou RH, Eckhart $\mathrm{AD}$, Koch WJ. Darbepoetin alfa, a long-acting erythropoietin analog, offers novel and delayed cardioprotection for the ischemic heart. Am J Physiol Heart Circ Physiol. 2007;293(1):H60-H68.

43. Chu $\mathrm{T}$, et al. Cardiotoxicity associated with the tyrosine kinase inhibitor sunitinib. Lancet. 2007;370(9604):2011-2019. 